\title{
A qualitative study of postgraduate students' perceptions of virtual patients in emergency medicine tutorials
}

\section{Jacqueline I. Wilson}

Centre for Medical Sciences Education, Faculty of Medical Sciences, The University of the West Indies, Trinidad and Tobago

Correspondence: Jacqueline I Wilson, Centre for Medical Sciences Education, Faculty of Medical Sciences, The University of the West Indies, St. Augustine, Trinidad and Tobago. Email: jacqui.wilson@sta.uwi.edu

\begin{abstract}
Objectives: To investigate students' reactions to introducing virtual patients into a postgraduate emergency medicine curriculum.

Methods: Qualitative data from semi-structured interviews were subjected to a thematic analysis. A convenient sample of seven postgraduate doctors in training enrolled in the diploma program participated in the study.

Results: The results indicate that participants' reactions to including virtual patients in tutorials yielded six themes: (a) Virtual patients are game-informed learning; (b) A virtual patient tutorial is collaborative learning; (c) Virtual patient is an authentic activity; (d) Virtual patients encourage reflection; (e) Virtual patients encourage clinical reasoning; and (f) Integrate virtual patients into the curriculum.
\end{abstract}

Conclusions: The implication of this study is that the virtual patient is a significant learning resource in postgraduate emergency medicine. Virtual patients may be used for independent study or collaborative learning. The virtual patient tutorial was a positive learning environment free of risk to patients in which learners practiced clinical reasoning. Virtual patients were authentic in context and culture, but their navigation needs improvement. Future work includes exploring alternative virtual patient designs and using virtual patients for assessment.

Keywords: Virtual patient, computer assisted learning, computer based simulation, medical education, emergency medicine

\section{Introduction}

Clinical training in emergency medicine is customarily conducted by "practicing diagnostic, therapeutic, and procedural skills on live patients". ${ }^{1}$ Emergency medicine is particularly challenging and error-prone because multiple patients who present with all types of illness and injury have to be managed simultaneously and expeditiously, and decisions have to be made with time pressures and sometimes incomplete information. ${ }^{2}$ There is increasing dissatisfaction with practicing on patients and greater emphasis for patient safety. ${ }^{3}$ Learning by doing is no longer acceptable with invasive procedures and high-risk situations. ${ }^{4}$ Reznek and colleagues ${ }^{1}$ decry the inefficient, opportunistic, and unstructured nature of the live patient model of education with its lack of opportunities for learners to make mistakes. Training today's clinicians to manage emergency and disaster medicine requires effective and flexible strategies that address the immediate needs of learners. Therefore, alternative methods are needed to gain clinical knowledge and experience.

Simulation technologies provide "opportunities to practice rare and critical events, safe and controlled environments that eliminate risk to patients, enhanced visualization, and authentic contexts for both learning and assessment." ${ }^{\text {S }}$ Simulations in medical education replace or amplify real patient experiences by imitating real clinical encounters. ${ }^{6}$ Virtual patients (VPs), which are interactive computer simulations of real-life clinical scenarios, enable learners to take on the role of the physician, in which they take a history, conduct a physical exam, and make diagnostic and therapeutic decisions. ${ }^{7}$

Users have found VPs useful, ${ }^{8}$ engaging, ${ }^{9}$ realistic, ${ }^{10}$ and relevant to clinical training. ${ }^{11}$ VPs have produced significant 
gains in clinical knowledge and attitudes. ${ }^{11}$ Students' examination skills have been significantly improved after using VPs, and they have retained the knowledge and skills long after the teaching episode. ${ }^{12,13}$ VPs have been perceived to be as effective as using standardized patients for teaching some skills. ${ }^{14,15}$ However, VPs have been associated with generating less empathy than with standardized patients. ${ }^{16,17}$ VPs can provide a risk-free environment to learn, practice, and measure clinical reasoning skills. ${ }^{14,18}$ VPs can expose students to situations they might not ordinarily encounter. ${ }^{19}$

Unlike previous VP research whose participants were undergraduate students with no clinical experience; this study's participants were doctors with clinical experience seeking postgraduate training in emergency medicine. For the first time, VPs were implemented at this university. Therefore, it was not known how students would react to this new teaching/learning tool and whether it was useful in a postgraduate emergency medicine curriculum.

The purpose of this study was to determine if virtual patients provided a safe, authentic learning environment in which learners' knowledge and reasoning were enhanced. This study explored participants' reactions to introducing VPs into the tutorials of a diploma program in emergency medicine. The results of this research can inform how computer-based simulations can be effectively used in medical education, and more specifically, to help physicians acquire knowledge and reasoning skills.

\section{Methods}

\section{Design}

Qualitative research approaches emphasize the presentation of differing perspectives and are most appropriate to answering research questions that focus on what happens in a given context. ${ }^{20}$ Qualitative methods allowed this researcher to identify, analyse, and report patterns in the learning experiences of one group of doctors seeking postgraduate training in emergency medicine during tutorials using virtual patients (VPs). Qualitative data from semi-structured interviews provided the researcher with insights into the learning environment in which VPs were used. Thematic analysis of this data yielded the outcomes of using VPs in a tutorial setting.

\section{Participants}

The University of the West Indies delivers an emergency medicine diploma program at its Trinidad campus. The aim of the 18-month part-time program is to equip clinicians with the core knowledge required to provide safe and effective emergency medical care in a variety of clinical settings. Students include both junior doctors wishing to specialize in emergency medicine, and senior doctors with significant prior experience in and a clinical commitment to emergency medicine. The program's instructional strategies comprise didactic lectures, student-led tutorials, problem based learning, group projects, journaling, case presentations, skills training, simulated patients, life support courses, role playing, and self-directed learning.

The participants were a convenient sample of 7 of the 12 students enrolled in the program in 2009. Interviews were conducted after the students' final exams for the diploma program. The remaining 5 students did not take their final exams during this period owing to illness or academic ineligibility. All participants were 21 years of age or older. The amount of time they spent practicing medicine ranged from 3 to 29 years. Their experience working in emergency departments ranged from 16 months to 24 years. Specialist training among the participants included general medicine, critical care, internal medicine, orthopaedics, and general surgery. Qualitative studies usually have a small sample size ${ }^{21}$ which permits gathering in-depth information. One qualitative technique is sampling to the point of redundancy; one can stop sampling when no more information is forthcoming. ${ }^{22}$ Redundancy was achieved after a convenient sample of 7 participants was interviewed.

\section{The virtual patients}

Four low-fidelity branching narrative VPs were created for this study using Open Labyrinth. Labyrinth was developed at the University of Edinburgh and is now freely available as an open-source web-based authoring and delivery application at Source Forge (https://sourceforge.net/projects/open labyrinth). The VPs were stored on a remote server that was accessed online via the World Wide Web. The VPs were made accessible to students through the university's online course management system (CMS). The CMS was password-protected and accessible only to students registered in the course. The VPs covered the topic of adult medical emergencies.

\section{Procedure}

Ethical approval was obtained from the university through the faculty's ethics committee before proceeding with this study. The participants were adult normal volunteers drawn from the postgraduate program in emergency medicine. Before any data was collected, the researcher had explained the purpose, nature, and proposed benefits of the study, and answered any questions on data security, anonymity, and confidentiality. Participants were then asked to read and sign an informed consent form. Any participant could have chosen to opt out of the study at any time, but none of them did. Students were introduced to VPs in a tutorial setting, in which the tutor asked students to manage the VP and justify the choices they made. In the tutorial setting, students had to come to a consensus on what choice to make and explain the reasoning behind their choices. Four tutorials were conducted, each lasting 30 to 50 minutes. All students were not always present at the start of the tutorials or for all four 
tutorials. As few as 6 students and as many as 12 students were present during the tutorials. After the tutorials had been completed, participants were interviewed using a guide comprised of both structured and open-form questions. This researcher designed the first five questions to gather information on participants' emergency medicine experience and motivation to study emergency medicine. The remaining questions were designed to gather opinions on the effectiveness and quality of VPs, the teaching and learning experiences with VPs, and open-ended comments. These remaining questions were based on questions taken from a published evaluation instrument ${ }^{23}$ designed specifically by the VP community to gather evidence to guide the design and integration of VPs. During the interviews, the researcher did not limit herself to these questions, their order, or content. Further questions were employed by the researcher as needed during the interview in order to obtain additional information. The interviews were recorded and transcribed.

Participants' names were replaced with numbers (for example Student1, Student2, etc.) to maintain privacy and confidentiality during transcription of recorded sessions and presentation of results. No participant expressed discomfort in being filmed nor did anyone state that he/she was inconvenienced by taking part in the interview. During the interview, the researcher explained that the participant had the right not to answer a question. Participants were assured that their comments would remain confidential and available only to the researcher, and their identities would not be disclosed.

\section{Analysis}

Interviews were treated as one data set and subjected to an inductive thematic analysis. ${ }^{24}$ The data was subjected to repeated readings; notes made during data collection were reviewed; notes were studied for meanings; data was reread and coded; a draft summary was written; the summary was reviewed with participants; and the final summary incorporated new interpretations. Constant comparisons between the emerging themes, themes existing in the literature, and the original data were made in order to ensure a good fit between data and findings.

An important limitation to recognize is the researcher of this study: her abilities, biases, and experiences. Qualitative findings may be subject to several interpretations. Personal experiences of the researcher in designing virtual patients and personal biases related to their use may be reflected in her communication. To minimize personal biases during data analysis, this researcher read the literature comprehensively and stayed open to alternative explanations. In keeping with the qualitative tradition, after analysing interviews, this researcher checked with participants that the writings reflected their perspectives.

\section{Results}

Six themes were identified from the thematic analysis of data gathered to explore participants' reactions to using virtual patients (VPs) in a tutorial setting: (a) VPs are gameinformed learning; (b) A VP tutorial is collaborative learning; (c) VP is an authentic activity; (d) VPs encourage reflection; (e) VPs encourage clinical reasoning; and (f) Integrate VPs into the curriculum. Each theme is explained and supported with quotes from the interviews. The quotes are labelled with fictive student interview numbers. Other students may have expressed the same idea in other words, but these were not included in order to avoid redundancy.

VPs are game-informed learning. When participants were asked to describe their experiences with and how they felt about VPs, they mentioned characteristics associated with game play. They thought VPs were engaging, motivating, interesting, enjoyable, exciting, stimulating, interactive, and it made learning fun and easy.

\section{I enjoyed them. Enjoyable! I would like more as a learning experience, I think it's a good one. (Student 5)}

\section{So, I found it fascinating, interesting, you know. And the beautiful thing about it was, it got the whole class, we were all interacting. Everybody like, had an opinion, what to say, what to do. I loved the fact that it was interactive. (Student 1)}

...it made going through a patient scenario easy, almost fun. It will never be fun, because is work. But it was a lot easier than say, how do you treat MI [myocardial infarction], and read a book on how to treat MI. If you didn't know before, coming out from the virtual patient, you would have a good grasp of and with very little effort....It was just easy to internalize. (Student 2)

The characteristics of multiple paths, navigation, feedback, choices and consequences, which are also associated with game play, were mentioned with respect to virtual patients. Taking different paths and having options were central to the success of the virtual patient. Participants recognised that it was not possible to have unlimited options, but they felt that there should never be a page without choices.

\section{And I loved the fact that you could go either way. I loved the options that you had. (Student 1)}

Interviewer: What were the best things about learning with online VP cases? Student 5: With the VP can explore options without harm to the patient. Still it is a learning tool. Interviewer: What were the worst things about learning with online VP cases? Student 5: Options that may not be given in the particular question. 
The only thing I noticed a little bit when you come lower down in the Virtual Patient, for example you have Virtual Patient, and what you want to do next? And you have X-ray and CT scan and you get an ultrasound, and you get ABGs and whatever, whatever [drawing]. Now if you choose now here, this, X-ray, then you will see that in the next ...now only these are left [showing few choices]... [until] only one is left. Now of course, that is hardly giving me a choice anymore because I know now I have to click this, I have to do the ABG. It's a little bit so, perhaps to stamp in my mind that at least I have to do the $A B G$ too, but since there are no choices anymore, now you taking almost over what I have to do... So that I think that at the end of the case, you should still introduce some other options again so that it still remains open. And if now of course, I'm going in the complete wrong direction, then you can correct me, via your feedback. But I simply should never have that question that gives me just one answer. (Student 3)

There was some feeling that navigating through some choices needed some improvement.

... a few times when you came to the end of ...let's do all these things, the program didn't move on to the next phase by itself. You then had to select something to go back and then select the thing to go back on. So if the program could somehow mark off your choices.... And so every time you chose one, the next menu that come up would have four things, but the program didn't keep a memory of what I chose before. So that at the end I would still have to go back and backtrack on my algorithm and then choose a different path. (Student 2)

There was also the feeling that participants would have liked to continue on with errors detrimental to the patient rather than being corrected.

\begin{abstract}
Also I found that if you went down the wrong path, it would have been exciting and interesting to continue leading the person down that path as opposed to bringing them back, so that they would see in a virtual setting that they could kill somebody. So it would have been exciting to have cases where the patient actually died and you could not go back to undo or re-choose another option after you made a mistake...., 'cause in real life you don't get to go back if you make a life-threatening error. And I believe it's better to learn from those errors in a virtual patient than in real life. (Student 4)
\end{abstract}

A VP tutorial is collaborative learning. When participants were asked to describe their experiences with and how they felt about VPs, characteristics associated with collaborative learning emerged from the analysis. Learning was viewed as a social activity with the opportunity to talk and share diverse perspectives. There was general consensus that there was much to be learned from one's peers.
We also had an opportunity to, just like I said earlier on, to listen to what other people. Because, you have an option, right? And it doesn't just end there, because someone comes up with another idea that may not be in the case. And you find it interesting. You learn. The fact that this guy comes in and says no, you shouldn't have done this and this would have been better if you had done it this way. And someone comes up with a similar situation that he had managed in the past and he comes up with what he did and it worked for him and we pick it up, which is something you would have missed if you had been doing it on your own. (Student 1)

We certainly had in our class, people coming from different sort of settings, some with more expert experience in other specialties as well. One of the guys I think he did anesthetics and that sort of thing. From their experiences too, a wealth of experience, from their experiences too we can learn. (Student 5)

In a group setting you get to hear the thoughts and opinions of others and get to have a nice debate. So, it's more interactive and more educational as a group. On your own, it's more of a kind of assessment than anything. (Student 4)

There was also agreement that, in a group setting, learning was affected by the dynamics of the group and the role one took in a group.

You see in groups, it depends if you're a talker or not a talker, I'm not really a talker. If I'm one-on-one I will talk but when I'm in a group, a large group, people just jump in before I even get a chance to say, what, so I tend or it comes across like, ok, I'm not saying anything but they may be saying things I wanted to say but they just said it faster than I did. I think you lose that sort of experience, I mean to get the individual to really, especially if you're not a person who just, you know, how should I put it...I don't want to use the word assertive but there are people like that, they just go into an area and they just take over. They do well in group sessions. People like myself who tend to be a little more reserved and you know, wait a little bit, and probably don't look like we do so well in those sessions. So l like the one-onone, even if I have to talk to one person and we go through. (Student 5)

You have the dominant persons and you have the...not so outgoing persons....Someone who is not as dominant in the group may not get to voice their opinions or ask questions as to why someone else chose something and just move on without addressing that person's concern. So that's the only problem with group dynamics. (Student 4 )

The participants felt that the role of the coach/mentor was paramount to guide and sometimes take charge of the learning process. The tutor challenged them to justify their choices by sharing their reasoning. 
The teacher sort of likes coordinates or moderates.... [If] a topic comes up and you are not very familiar with, which...you couldn't do it right there in the class, and all you have going is your colleagues' opinions, which could be wrong or right. But that's where the lecturer comes in, or the moderator comes in, to put you in the right. (Student 1)

Virtual patient is problem oriented teaching because you start with the problem and you deal with the problem, whatever. In my limited experience then of course in this diploma program that I found that the problem oriented teaching, it depends on the moderator... (Student 3 )

All participants said that VP should be done both as an individual activity and in a group. However, there was no consensus on which activity should come first.

I would insist that everybody does it on his own, and then we do it again together as a group. And then explain to each other why we made this choice and why we didn't make that choice. So the group session is more of a feedback mechanism, while the individual is in the first place to shape your thinking and decision-making process. (Student 3 )

It's good to hear what other people think may be best and why. Even if the virtual patient say that we should do $A$ and somebody in class say, well you know, you would say that and in most cases it could be so, but then you might learn something extra when you do it in a group....For me when I do a virtual patient at home, I learn what the virtual patient wanted to teach me, so I could learn that. But now, I could learn from the virtual patient and other people. (Student 2)

It takes the fun out of it actually when you do it on your own. It does actually because we also had the opportunity to do it on our own when we were revising, but I realize it wasn't as much fun. Because you want to know other people's opinions....It's good when you're revising to do it on your own, but initially, it's usually best to do it in a group. (Student 1)

VP is an authentic activity. When participants were asked to describe their experiences with and how they felt about VPs, they mentioned characteristics associated with authentic activities. They thought VPs were realistic, relevant, and developed for the local environment. They felt that they were facing people and situations they would see in practice and reacting as they would in the emergency department.

They sounded like real live patients that we see every day in the emergency room. And we find we were saying things that we ordinarily would have done if we had such a patient in front of us. Those patients were patients that we could relate to, like patients that we are accustomed to seeing. (Student 1)
I like the names they used and the ethnicity and the kinds of conditions that would come in and the patient layman's terms and so on, that was a nice thing to see. (Student 4)

VPs encourage reflection. When participants were asked to describe their experiences with and how they felt about VPs, they mentioned characteristics associated with reflection when they were reviewing VPs on their own. On their own, they had the opportunity to review the content, to make different choices and correct mistakes, to do research, and to realize the importance of VP for learning.

When you are alone you can focus even more on it and maybe if you take your time with it, because that's the next thing, you can take a lot of time with it and if you don't understand something, you can sit down with your books and research at the same time, so it was a learning experience, one-on-one, it all depends on how we process the information. Some people love chatting it out and they learn by that experience, some people have to read and read and reread. (Student 5)

\begin{abstract}
...it's not that when I was in a group, I couldn't concentrate, but I was at home doing this, I can look into it more, what is the importance of it, you understand when you are alone. When you do the mistakes, when you're alone, you correct it and you try to learn more. (Student 7)
\end{abstract}

VPs encourage clinical reasoning. All participants agreed that their clinical reasoning and diagnostic skills were enhanced by working through the VPs. After completing the VPs, they felt better prepared to care for real patients with similar complaints. They all agreed that they had to collect and integrate information to diagnose and manage the patient and make the decisions that affected the patient. When participants were asked to describe their experiences with VPs, they mentioned characteristics associated with analytic reasoning. Analytic reasoning involves learning the rules that link signs and symptoms to diagnoses. ${ }^{25}$ They were ordering their thoughts, proceeding in a stepwise fashion, and refining their mental models.

I felt it was excellent because it helped me a lot to treat the patient in a stepwise fashion without confusion. (Student 6)

One thing the virtual patient does and does well, it reinforces protocols. So not just the end result, then, but steps to get the end result. The virtual patient will reinforce that very well. This is your priority. Do this next, do that next, not just, well at some point in time you should probably get a [n] ultrasound. It reinforces that they have appropriate times to do stuff. (Student 2) 
The virtual patient gives you a set amount of answers and then you have to choose the right one. In doing so, sometimes it might stimulate that part of your brain and say, oh, that looks right. So you choose, or it might stimulate some information in the deep processes and you get the answer that way....The virtual patient, you [are] literally being given answers and you have to choose one. Maybe that may not be as good, if you want to really want to stimulate the brain to get the answer, but it is good in fact that it helps to teach you to order your thought. (Student 5)

Integrate VPs into the curriculum. The course instructor had introduced VP tutorial as a patient-oriented teaching strategy that was not previously available within the program. All participants in this study claimed that VPs were new to them, "[they] had never had that experience before" (Student 6). Participants claimed that VP needed to be integrated into their courses and not just used as add-on, because VP "has potential to be a really significant teaching aid" (Student 4) and "integration of VP cases...would make learning more fun and ... easier.” (Student 1)

\begin{abstract}
I think it started off on a good footing. The only thing is that we didn't have it enough, you understand. We only had a few and those few felt like experiments. They didn't feel like part of the course. They should feel like part of it. So we still have to internalize it, integrate it into our system. (Student 1)
\end{abstract}

\section{Discussion}

This study was embedded in a postgraduate diploma program for clinicians with significant emergency workload. There was a range of age, experience, specialist training, and country of origin among the participants. Most VP studies used undergraduate students with little clinical experience. ${ }^{26}$ This study used participants familiar with the clinical context who were expected to be more knowledgeable and self-directed.

VPs are game-informed learning. Games are exciting, engaging, and compelling, and encourage individuals to attempt difficult tasks repeatedly. ${ }^{27}$ In game-informed learning, the principles of successful game play are applied to active learning approaches so that education becomes more playful. ${ }^{28}$ As in previous studies, this study's participants held perceptions of VP that had characteristics associated with game play. ${ }^{9,29}$ Begg and colleagues ${ }^{27}$ consider the characteristics of successful game play to be similar to successful learning. The player or learner has goals, appreciates challenge and interaction while developing successful practices. Begg ${ }^{28}$ recommends making education more game-like by having activities containing a task or challenge for the learner who has to adopt an active, consequential role in an environment designed for interaction and exploration. He adds that scores, conditional blocks, countdowns, and randomizations are beneficial. In this study, although there was evidence of using VPs for exam preparation, there was no evidence of repeated use of the same VPs. It is not known whether students would repeatedly use the same VP without additional options or increased complexity. The question remains whether it is more beneficial to develop more VPs or more (complex) variations on existing VPs.

A VP tutorial is collaborative learning. A review of VPs in health professions education concluded from the qualitative research that working in groups is important. ${ }^{26}$ During collaborative learning, students are expected to interact and work together on learning tasks to achieve a common learning goal. ${ }^{30,31}$ The students did not have different roles during the activity, and there was no division of labour. Together they were expected to diagnose and manage their VP. Learning was viewed as a social activity with the opportunity to talk and share diverse perspectives. Socio-cultural learning theories offer a best-fit explanation of learning in clinical contexts. ${ }^{32}$ Knowledge is co-constructed and distributed across and among people, processes, and the cultural artefacts of a community of practice. ${ }^{33} \mathrm{Co}-$ construction of clinical knowledge occurs because of interactions among peers, and through diagnostic tools that mediate the action. ${ }^{34}$

According to Panitz, ${ }^{35}$ collaborative learning has academic, social, and psychological benefits. Collaborative learning promotes critical thinking and higher achievement, models problem-solving techniques, creates a social support system for students, encourages diversity understanding, establishes a positive atmosphere for cooperation and helping, increases students' self-esteem, and promotes positive attitudes. One of the challenges to collaborative learning is loss of control in managing the group process. Participants agreed that there was much to be learned from one's peers. There was also agreement that in a group setting, learning was affected by the dynamics of the group and the role one takes in a group. It was observed during this study that when some students were boisterous in behaviour, other students would discuss together quietly. However, during interviews, all participants agreed that VP should be used in both an individual and a group setting.

The role of the tutor. There is a central place for the cli-

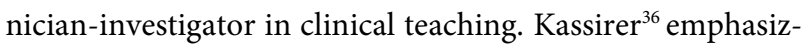
es the importance of a coach in teaching clinical reasoning. The coach monitors learning, ensures participant interaction, and provides feedback. The participants in this study felt that the role of the tutor was paramount to guide and sometimes take charge of the learning process. Kassirer ${ }^{36}$ recommends that the coach be unfamiliar with the case and so has to examine the information as the learners do. However, Kirschner and colleagues ${ }^{37}$ posit that strong instructional guidance is superior to discovery learning and that unguided instruction may have negative results when students have misconceptions or disorganized knowledge. Sandars ${ }^{38}$ also supports the claim that guided reflection is important to reveal underlying beliefs and assumptions. In this study, the tutor invited participants to elaborate on 
their knowledge structures through his use of open questions. The tutor prevented participants from premature closure by prompting them to gather more data, and to consider alternative hypotheses. Dornan and colleagues ${ }^{39}$ found that students using a web-based learning management system were most active and motivated when supported by clinical teachers. In this study, the tutor managed the learning environment, while providing affective and pedagogic support.

VP is an authentic activity. According to Brown and colleagues, ${ }^{40}$ authentic activities are what experts within a community do when engaged with real-world problems. Authentic activities reflect the natural complexity of the real-world environment. All of the participants felt that the VPs were based on real patients and that they were required to perform as they would in a clinical context. This finding is supported by previous authors ${ }^{9}{ }^{41}$ who also found VP to be authentic or realistic. This study is unlike that of Kiegaldie and White ${ }^{41}$ in which the feeling of realism came from sounds and images. This study is akin to Zary et al. ${ }^{9}$ in which the feedback provided by VP added to the realism.

Brown and colleagues ${ }^{40}$ warn of classroom activities that form part of class culture and hence would not reflect the clinical context. The findings of this study support the VP tutorial as a form of authentic learning that exposed the uncertainty in real-life decision making. Participants felt that VP provided an authentic representation of clinical context. The content, language, clinical data, and design of the VPs in this study, which included alternative routes, were crucial to presenting an authentic experience. The VPs used in this study were developed locally from real patient cases. Fors and colleagues ${ }^{42}$ identify the following cultural issues that impact on the use, development, and acceptance of VPs: (a) ethnicity - some medical conditions are more frequent in some ethnic groups and may present differently among groups, (b) language - patients may use local words and expressions to describe their problem, and (c) socioeconomic - countries may differ in medical procedures, regulations, and resources for healthcare. When VPs reflect local conditions, users consider the VPs more authentic and are more engaged in them.

Authentic learning should also help students develop expertise in the conative domain of acting, deciding, and committing. ${ }^{43}$ In this study, as in a previous one, ${ }^{29}$ participants believed one of the advantages of VP was that it allowed users to make decisions, and when VP was used for self-study, one's decision making skills and confidence were reinforced.

VPs encourage reflection. An effective way of learning in professions such as medicine is through reflection. ${ }^{44}$ Boud, Keogh and Walker ${ }^{45}$ defined reflection as "a generic term for those intellectual and affective activities in which individuals engage to explore their experiences in order to lead to a new understanding and appreciation". Participants claimed to have engaged in reflection, especially when they used VPs for self-study. Providing feedback supports the reflective process. Reflective elements built into the VP that prompted reflection were: allowing the user to review choices and providing feedback. Feedback allowed the user to consider the consequences of their actions. Guided reflection by a mentor is particularly important in professional practice. ${ }^{38}$ The mentor facilitates reflection, while supporting and challenging the learner's beliefs and assumptions. This study supports previous findings that reflective practice can be taught and improved when carried out within a safe atmosphere, with mentorship and supervision, peer support and time to reflect. ${ }^{46}$

VPs encourage clinical reasoning. The results of this study demonstrate a preliminary acceptance of these VPs for enhancing clinical reasoning. These findings are concurrent with previous research. ${ }^{47}$ Participants agreed that their clinical reasoning and diagnostic skills were enhanced by working through VPs, and they felt better prepared to care for real patients with similar complaints. Both analytic and non-analytic reasoning processes should be promoted in clinical teaching. ${ }^{25}$ Participants described their experiences with VPs in terms of characteristics associated with analytic reasoning. VP provided structure and feedback, which helped them order their thoughts, proceed in a stepwise fashion, and refine their mental models. VP corrected them, prioritized information, and reinforced protocols and what they knew.

Integrate VPs into the curriculum. There was a general consensus that VPs needed to be integrated into the curriculum in order to be an effective teaching/learning tool. Using VPs for independent study and collaborative learning were both endorsed as effective teaching/learning strategies. It was not clear if VP should be used to teach new material or to reinforce what had been learned. It was also not clear whether more VPs or more (complex) variations on existing VPs should be developed.

In conclusion, a positive learning environment free of risk to patients was created in which learners practiced clinical reasoning. Participants were satisfied that the branching narrative VPs created for this study were authentic, reflected local conditions, and were of a high quality, although some participants felt that the navigation could be improved. The results support previous research related to acceptance of VPs and integrating e-learning activities. ${ }^{48,49}$ The results of this study also suggest that branching narrative VPs designed for the local setting can be effectively used in a tutorial by participants with clinical experience to promote clinical reasoning. It can be concluded that VP has the potential to be a significant teaching/learning tool.

\section{Limitations of the study}

The small, convenient sample of participants limited the generalizability of the study results. Another limitation was that only the perceptions of students who chose to participate were included. During the interviews, participants' 
responses may have been biased due to the presence of the researcher. The researcher's abilities, biases, and experiences were also important limitations that may be reflected in the communication. Although this research study was limited to one convenient sample of students in one medical school, in one multiethnic and multicultural country, its purpose, findings, conclusions, and recommendations should be of interest to the community of medical educators.

\section{Future considerations}

The immediate considerations are to create more virtual patients, to improve the navigation so that users cannot go backwards, and to integrate the cases into the postgraduate emergency medicine curriculum by incorporating them in all modules of the program. Alternative virtual patient designs should also be explored. A further consideration is to consider how virtual patients can be used for formal assessment.

There is a need for greater understanding of how computer-based simulations impact student motivation, learning, and ultimately, patient care. Future research could explore student preferences and performance when virtual patients are used for self-study versus use in a collaborative environment. They could also investigate student preferences for and measure differences in motivations, knowledge, and reasoning with different virtual patient designs. Additional research would be beneficial to determine valid and reliable measures of using virtual patients for assessment.

\section{Acknowledgments}

This research was carried out in part fulfilment for the Degree of Doctor of Education. Thanks go to St. George's University of London for web hosting our virtual patients and to the participants within the study who shared their thoughts and experiences. Ethical approval was obtained from the University of the West Indies and Northcentral University.

\section{Conflict of Interest}

The authors declare that they have no conflict of interest.

\section{References}

1. Reznek M, Harter P, Krummer T. Virtual reality and simulation: Training the future emergency physician. Acad Emerg Med. 2002;9:78-87.

2. Cosby KS, Croskerry P. The nature of emergency medicine. In: Croskerry P, Cosby KS, Schenkel SM, Wears RL, editors. Patient safety in emergency medicine. Philadelphia, PA: Lippincott Williams \& Wilkins; 2009.

3. McLaughlin S, Fitch MT, Goyal DG, Hayden E, Kauh CY, Laack TA, et al. Simulation in graduate medical education 2008: A review for emergency medicine. Acad Emerg Med. 2008;15:1117-29.

4. Vozenilek J, Huff JS, Reznek M, Gordon JA. See one, do one, teach one: advanced technology in medical education. Acad Emerg Med. 2004;11:1149-54.

5. AAMC. Effective use of educational technology in medical education Colloquium on educational technology: Recommendations and guidelines for medical educators. Washington, DC: Association of American Medical
Colleges; 2007.

6. Gaba DM. The future vision of simulation in health care. Qual Saf Health Care. 2004;13:i2-10.

7. Ellaway R, Candler C, Greene P, Smothers V. An architectural model for MedBiquitous virtual patients. Baltimore, MD: MedBiquitous; 2006.

8. D'Alessandro DM, Lewis TE, D'Alessandro MP. A paediatric digital storytelling system for third year medical students: the virtual paediatric patients. BMC Med Educ. 2004;4.

9. Zary N, Johnson G, Fors U. Web-based virtual patients in dentistry: factors influencing the use of cases in the Web-SP system. Eur J Dent Educ. 2009;13:2-9.

10. Courteille O, Bergin R, Stockeld D, Ponzer S, Fors U. The use of a virtual patient case in an OSCE-based exam - A pilot study. Med Teach. 2008;30:e66-e76.

11. Sanders CL, Kleinert HL, Free T, King P, Slusher I, Boyd S. Developmental disabilities: improving competence in care using virtual patients. J Nurs Educ. 2008;47:66-73.

12. Vukanovic-Criley JM, Boker JR, Criley SR, Rajagopalan S, Criley JM. Using virtual patients to improve cardiac examination competency in medical students. Clin Cardiol. 2008;31:334-9.

13. Frisby AJ, Lane JL, Carr AM, Ross E, Gottlieb RP. Development and evaluation of an interactive multimedia clinical skills teaching program designed for the pediatric clerkship. Teach Learn Med. 2006;18:18-21.

14. Stevens A, Hernandez J, Johnsen K, Dickerson R, Raij A, Harrison C, et al. The use of virtual patients to teach medical students history taking and communication skills. Am J Surg. 2006;191:806-11.

15. Triola M, Feldman H, Kalet AL, Zabar S, Kachur EK, Gillespie C, et al. A randomized trial of teaching clinical skills using virtual and live standardized patients. J Gen Intern Med. 2006;21:424-9.

16. Deladisma AM, Cohen M, Stevens A, Wagner P, Lok B, Bernard T, et al. Do medical students respond empathetically to a virtual patient? Am J Surg. 2007;193:756-60.

17. Harter LM, Kirby EL. Socializing medical students in an era of managed care: the ideological significance of standardized and virtual patients. Communication Studies. 2004;55:48-67.

18. Zary N. Virtual patients for education, assessment and research: A webbased approach [Ph.D thesis]. Stockholm, Sweden: Karolinska Institutet; 2007.

19. Critchley LAH, Wong JWY, Leung JYC. Virtual patients and undergraduate anaesthesia teaching. Med Educ. 2008;42:1120-1.

20. Patton MQ. Qualitative research and evaluation methods. 3rd ed. Thousand Oaks, CA: Sage; 2002.

21. Gall MD, Gall JP, Borg WR. Educational research: An introduction. 8th ed. Boston, MA: Pearson Education/Allyn \& Bacon; 2007.

22. Lincoln YS, Guba EG. Organizational theory and inquiry: the paradigm revolution. Beverly Hills, CA: Sage; 1985.

23. Huwendiek S, Haider HR, Tönshoff B, de Leng BA. Evaluation of curricular integration of virtual patients: Development of a student questionnaire and a reviewer checklist within the electronic virtual patient (eVIP) project. Bio-Algorithms and Med-Systems. 2009;5:35-44.

24. Braun V, Clarke V. Using thematic analysis in psychology. Qualitative Research in Psychology. 2006;3(2):77-101.

25. Eva KW. What every teacher needs to know about clinical reasoning. Med Educ. 2005;39:98-106.

30. Lehtinen E, Hakkarainen K, Lipponen L, Rahikainen M, Muukkonen H. Computer supported collaborative learning: A review. 2001 [cited 2012 January 13]; Available from: http://www.comlab.hut.fi/opetus/205/etatehta val.pdf.

26. Cook DA, Erwin PJ, Triola MM. Computerized virtual patients in health professions education: A systematic review and meta-analysis. Acad Med. 2010;85:1589-602.

27. Begg M, Dewhurst D, Macleod H. Game-informed learning: Applying computer game processes to higher education. Innovate; 2005 [cited 2008 April 24];1(6). Available from: http://innovateonline.info/index.php?view= article\&id $=176$.

28. Begg M. Leveraging game-informed healthcare education. Med Teach. 2008;30:155-8. 
29. Poulton T, Conradi E, Kavia S, Round J, Hilton S. The replacement of 'paper' cases by interactive online virtual patients in problem-based learning. Med Teach. 2009;31:752-8.

31. Yew EHJ, Schmidt HG. Evidence for constructive, self-regulatory, and collaborative processes in problem-based learning. Adv Health Sci Edu. 2009;14:251-73.

32. Bleakley A. Broadening conceptions of learning in medical education: the message from teamworking. Med Educ. 2006;40:150-7.

33. Lave J, Wenger E. Situated cognition: Legitimate peripheral participation. Cambridge: Cambridge University Press; 1991.

34. Radomski N, Russell J. Integrated case learning: teaching clinical reasoning. Adv Health Sci Edu. 2010;15:251-64.

35. Panitz T. The case for student centered instruction via collaborative learning paradigms. 2001 [cited 2012 January 13]; Available from: http://home.capecod.net/ tpanitz/tedsarticles/coopbenefits.htm.

36. Kassirer JP. Teaching clinical reasoning: case-based and coached. Acad Med. 2010;85:1118-24.

37. Kirschner PA, Sweller J, Clark RE. Why minimal guidance during instruction does not work: an analysis of the failure of constructivist, discovery, problem-based, experiential, and inquiry-based teaching. Educ Psychol. 2006;41:75-86.

38. Sandars J. The use of reflection in medical education: AMEE Guide No. 44. Med Teach. 2009;31:685-95

39. Dornan T, Hadfield J, Brown M, Boshuizen HPA, Scherpbier AJJA. How can medical students learn in a self-directed way in the clinical environment? Design-based research. Med Educ. 2005;39:356-64.

40. Brown JS, Collins A, Duguid P. Situated cognition and the culture of learning. Educational Res. 1989;18:32-42.

41. Kiegaldie D, White G. The virtual patient - development, implementation and evaluation of an innovative computer simulation for postgraduate nursing students. JEMH. 2006;15:31-47.

42. Fors UG, Muntean V, Mihaela B, Zary N. Cross-cultural use and development of virtual patients. Med Teach. 2009;31:732-8.

43. Lombardi MM. Authentic learning for the 21st century: an overview. Educause; 2007 [cited 2007 September 27]; Available from: http://www.educause.edu/ir/library/pdf/ELI3009.pdf.

44. Schön DA. Educating the reflective practitioner. San Francisco, CA Jossey-Bass; 1987.

45. Boud D, Keogh R, Walker D. Reflection: turning experience into learning. London: Sage; 1985.

46. Mann K, Gordon J, MacLeod A. Reflection and reflective practice in health professions education: a systematic review. Adv Health Sci Edu. 2009;14:595-621.

47. Gesundheit N, Brutlag P, Youngblood P, Gunning WT, Zary N, Fors U. The use of virtual patients to assess the clinical skills and reasoning of medical students: initial insights on student acceptance. Med Teach. 2009;31:739-42

48. eViP. Case studies of the use of virtual patients. London: St George's University of London; 2008 [cited 20092 February]; Available from: http://www.virtualpatients.eu/wp-content/uploads/2008/03/evip_deliverabl e_42_final.pdf.

49. Hege I, Ropp V, Adler M, Radon K, Masch G, Lyon H, et al. Experiences with different integration strategies of case-based e-learning. Med Teach. 2007;29:791-7. 